\begin{tabular}{|c|c|c|c|c|}
\hline & Number & $\begin{array}{l}\text { (\%) Declined } \\
\text { treatment }\end{array}$ & $\begin{array}{l}\text { Lost to } \\
\text { follow-up }\end{array}$ & $\begin{array}{l}\text { Stopped due to } \\
\text { side-effects }\end{array}$ \\
\hline Contact screening & $\begin{array}{l}105 \\
(50.9)\end{array}$ & 1 & 8 & 8 \\
\hline Biological therapy & $\begin{array}{l}71 \\
(34.4)\end{array}$ & 1 & 0 & 2 \\
\hline $\begin{array}{l}\text { New entrant screening } \\
18(8.7)\end{array}$ & 1 & 0 & 0 & \\
\hline Occupational health & $12(5.8)$ & 0 & 0 & 0 \\
\hline $\begin{array}{l}\text { Total number of } \\
\text { patients seen }\end{array}$ & 206 & 3 & 8 & 10 \\
\hline
\end{tabular}

\section{P118 HOW DO FOREIGN-BORN PATIENTS WITH TUBERCULOSIS ACCESS HEALTHCARE? A COHORT ANALYSIS OF REFERRALS FROM GENERAL PRACTICE AND THE EMERGENCY DEPARTMENT TO A TERTIARY TUBERCULOSIS SERVICE}

${ }^{1} S$ Conway, ${ }^{1} \mathrm{~A}$ Pitcher, 'S Dart, ${ }^{2} \mathrm{D}$ Vaghela, ${ }^{2} \mathrm{MGK}$ Burman, ${ }^{2} \mathrm{f}$ Potter, ${ }^{1} \mathrm{VLC}$ White, ${ }^{1} \mathrm{~S}$ Tiberi, ${ }^{2} \mathrm{H}$ Kunst. ${ }^{1}$ Barts Health NHS Trust, London, UK; ${ }^{2}$ Queen Mary, University of London, London, UK

\subsection{6/thoraxjnl-2016-209333.261}

Introduction More than Seventy percent of active Tuberculosis (TB) cases in England are in patients born outside the United Kingdom (UK). Lack of access to primary healthcare is often cited as a barrier to TB control. We considered how patients with TB referred directly to outpatient services initially access healthcare. Method A retrospective cohort analysis of all patients with active TB on the London TB register (LTBR) between April 2014 and April 2015 at a large urban tertiary referral centre. The route of referral to TB services was confirmed by a review of electronic patient records. We compared demographic, disease and outcome variables between groups as recorded in the LTBR. We excluded those requiring admission; identified through contact tracing; referrals from other secondary care outpatient services and those with inadequate data. Chi squared or Exact tests were used in the analysis.

Results We compared patients diagnosed with TB who were referred directly to outpatient services from General Practice (GP) (97 patients) and the Emergency Department (ED) (35 patients). There was no significant difference in age or sex between groups.

Of those patients born outside the UK (105), 78 percent (82/ $105)$ were referred to clinic from their GP compared to only 56 percent $(15 / 27)$ of those born within the UK (15/27). This difference was statistically significant $(\mathrm{p}<0.05)$. There was no statistically significant difference between the mean length of stay in the UK amongst migrants that presented via ED or GP (MD 2.33 years, $95 \% \mathrm{CI}$ : -2 to $7, \mathrm{p}<0.4)$. There was no statistically significant difference in the number of patients who had at least one social risk factor between groups.

Comparing disease between the groups, there was a higher proportion of multisite disease amongst those referred from ED compared to GP $(23 \%$ [8/35] vs $14 \%$ [14/97], p < 0.025), there was no statistical difference between the numbers of pulmonary cases identified or smear status between the groups.
Conclusion Amongst patients with active TB referred directly to outpatient services, those born outside the UK were more likely to have been referred by their GP than UK-born patients.

\section{P119 USING ADVERSE EVENTS IN A TUBERCULOSIS TRIAL TO DESCRIBE THE TOLERABILITY OF STANDARD THERAPY}

${ }^{1} \mathrm{CD}$ Tweed, ${ }^{1} \mathrm{G}$ Wills, ${ }^{1} \mathrm{AM}$ Crook, ${ }^{1} \mathrm{SK}$ Meredith, ${ }^{1} \mathrm{AJ}$ Nunn, ${ }^{2} \mathrm{CM}$ Mendel, ${ }^{2} \mathrm{SR}$ Murray, ${ }^{3} \mathrm{TD}$ McHugh, ${ }^{4} \mathrm{SH}$ Gillespie. ${ }^{1} \mathrm{MRC}$ Clinical Trials Unit at University College London, London, UK; ${ }^{2}$ The TB Alliance, New York, USA; ${ }^{3}$ Division of Infection and Immunity, University College London, London, UK; ${ }^{4}$ University of St Andrews Medical School, St Andrews, UK

\subsection{6/thoraxjnl-2016-209333.262}

Introduction and objectives The current standard treatment for pulmonary tuberculosis (TB) has been in use for several decades and the major risks associated with each of the four drugs (HRZE) are well recognised. However, large prospective trials with regular review and documentation of adverse events while taking HRZE are lacking.

We used the incidence of grade 3 and 4 adverse events (AEs) and serious adverse events (SAEs) in patients taking HRZE in the REMoxTB trial to investigate the overall tolerability of the regimen.

Methods Grade 3 or 4 AEs and SAEs (of any grade) for patients taking standard TB therapy were analysed. Events were labelled as occurring in the intensive phase, continuation phase or in follow-up (up to 18 months after enrolment). ANOVA and chi-

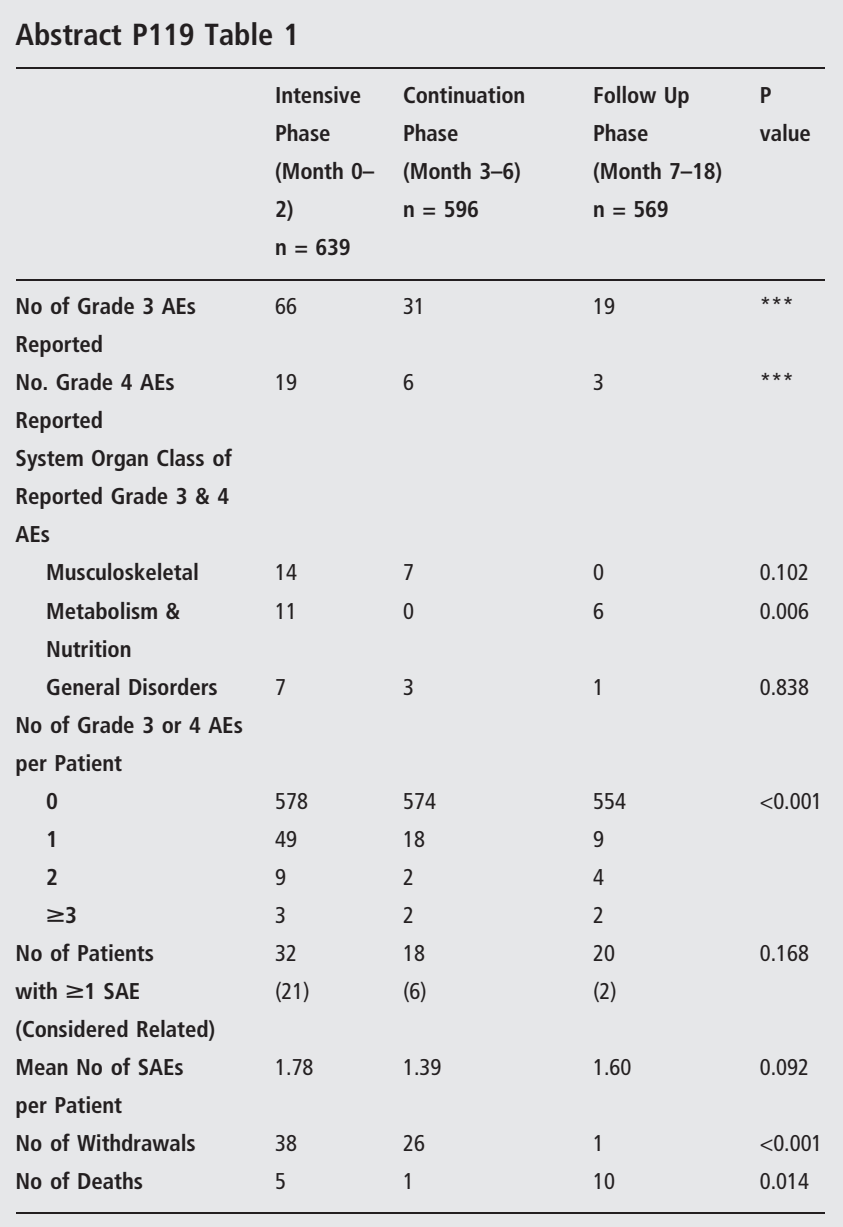


square testing was used to test for significant differences in the incidence of events across the treatment phases. Logistic regression was used to investigate associations between baseline characteristics and on-treatment SAEs and withdrawal from treatment, death or relapse/treatment failure.

Results 201 (31.5\%) of 639 patients taking standard therapy experienced grade 3/4 AEs or SAEs during treatment. AEs, SAEs, and withdrawals from treatment occurred most frequently in the intensive phase (see Table). Of 116 SAEs reported 84 (72.4\%) improved or resolved and were most commonly respiratory (16.4\%), gastrointestinal (6.9\%), and infection (5.2\%) related. There were 10 deaths in follow-up due to suicide, trauma, TB relapse, and acute illness. Logistic regression detected a significant association between on-treatment SAEs and withdrawal ( $p<0.001)$ or death $(\mathrm{p}<0.001)$, but not relapse/treatment failure $(\mathrm{p}=0.611)$. HIV-positive status (OR 4.25, $\mathrm{p}=0.016)$ and lower baseline weight (OR 1.46, $\mathrm{p}=0.023)$ were associated with the reporting of on-treatment SAEs.

Discussion AEs and SAEs were predominantly reported in the intensive phase, probably due to a combination of TB and effects of medication. However most deaths occurred in follow-up and were unrelated, emphasising the impact that social circumstances have on TB patients. The lack of significant association between SAEs during treatment and relapse/treatment failure provides reassurance that a complicated treatment period can end with successful treatment of TB. The association between SAEs on treatment and lower weights at baseline and HIV infection reinforces the need to monitor these patients closely.

\section{Clinical Studies of Asthma}

\section{P120 CHALLENGES IN USING HIERARCHICAL CLUSTERING TO IDENTIFY ASTHMA SUBTYPES: CHOOSING THE VARIABLES AND VARIABLE TRANSFORMATION}

\begin{abstract}
${ }^{1} \mathrm{M}$ Deliu, ${ }^{2} \mathrm{~S}$ Yavuz, ${ }^{1} \mathrm{M}$ Sperrin, ${ }^{3} \mathrm{D}$ Belgrave, ${ }^{2} \mathrm{C}$ Sackesen, ${ }^{2} \mathrm{U}$ Sahiner, ${ }^{3} \mathrm{~A}$ Custovic, ${ }^{2} \mathrm{O}$ Kalayci. ${ }^{1}$ University of Manchester, Manchester, UK; ${ }^{2}$ Hacceteppe University, Ankara, Turkey; ${ }^{3}$ mperial College, London, UK
\end{abstract}

\subsection{6/thoraxjnl-2016-209333.263}

Introduction The use of unsupervised clustering has identified different subtypes of asthma. Choosing the variables to input into the clustering algorithm is one of the important considerations. The majority of previous studies selected variables based on expert advice, whilst others used dimension reduction techniques such as principal component analysis (PCA). We aimed to compare the results of unsupervised clustering when using raw variables, or variables transformed using dimensionality reduction techniques.

Methods We performed our analysis on 613 asthmatics aged 623 years from Ankara, Turkey. We conducted extensive phenotyping and recorded 49 variables including demographic data, sensitisation, lung function, medication, peripheral eosinophilia, and markers of asthma severity. We performed hierarchical clustering (HC) using: (1) all variables; and (2) variables transformed using dimensionality reduction techniques.

Results PCA revealed 5 components describing atopy and variations in asthma severity, which were then used to infer cluster assignment. The optimal HC solution in both PCA-transformed and raw untransformed data identified five clusters. However, these clusters were not identical. Both identified mild asthma with good lung function, severe atopic asthma and late-onset mild atopic asthma. However, the overlap between children assigned to these three clusters in two HC analyses was modest. Clustering without PCA identified early-onset severe atopic asthma and late-onset atopic asthma with high BMI, whilst early onset non-atopic mild asthma in females was identified in HC with PCA. Using both methods, we identified four features that characterised the clusters. These were age of onset, atopy, asthma attacks, and asthma severity. Using only these four features, we identified early onset atopic mild asthma, early onset non-atopic mild asthma, severe asthma, late onset asthma, and exacerbation prone asthma. Cluster stability increased drastically.

Conclusion Different methodologies applied to the same dataset identified differing clusters of asthma. We identified four features that characterised the clusters. We propose that these four features could be more useful in identifying asthma endotypes.

\section{P121 URBAN FINE AND COARSE MODE PARTICULATE MATTER DIFFERENTIALLY ALTER THE MATURATION OF MONOCYTE-DERIVED DENDRITIC CELLS}

TR Ho, N Camina, PE Pfeffer, EH Mann, IS Mudway, CM Hawrylowicz. King's College London, London, UK

\subsection{6/thoraxjnl-2016-209333.264}

Background There is considerable evidence linking increased exposure to particulate matter (PM2.5 and PM10) to adverse respiratory outcomes, with patients with asthma and chronic obstructive pulmonary diseases (COPD) prone to more exacerbations and respiratory tract infections during pollution episodes. We have previously shown that peripheral human CD1c+ myeloid DCs, a key orchestrator of the adaptive immune response, promoted naive CD4 $+\mathrm{T}$ lymphocyte proliferation and increased expansion of potent inflammatory Th1 and Th17 effector cells when challenged with standard reference urban PM (SRM1648a). However, monocyte-derived dendritic cells (MDDCs), which represent an inflammatory subset of DCs, may play a substantial role in lung inflammation. In this study, we investigated the effects of PM on MDDC maturation, focusing on responses to PM samples collected from the contemporary London airshed. Methods PM2.5 and PM10 from a high traffic roadside site in London were collected $(2 / 1 / 2013$ - 15/1/2014) and pooled to generate representative annual samples. SRM-1648a and SRM2975 (diesel exhaust derived PM) were used as control samples. Differentiated MDDCs, derived from CD14+ monocytes incubated with IL-4 and GM-CSF for 6 days, were challenged with these PM samples, plus control PM samples at various concentrations $(2.5-20 \mu \mathrm{g} / \mathrm{ml})$ for 24 hours. DC expression of CD83, MHC-I and MHC-II were measured by flow-cytometry.

Results Roadside PM10 and SRM-1648a exposure significantly increased the expression of MHC-II on MDDCs, whilst Roadside PM2.5 and SRM-2975 had no impact. There was also an increased expression of maturation marker CD83 when cells were exposed to Roadside PM10 and SRM-1648a, as well as Roadside PM2.5 at a higher concentration. However, exposure to all PM samples except for Roadside PM10 led to a significant decrease in MHC-I expression.

Conclusion PM fractions containing coarse mode material (SRM1648a and PM10) appeared more able to stimulate MDDC maturation than fine mode PM (SRM-2975 and PM2.5). There was a clear decrease in MHC-I expression after exposure to most PM samples. This may indicate that MDDCs DCs exposed to PM are less able to stimulate $\mathrm{CD} 8+\mathrm{T}$ cells, resulting in recurring 